\title{
Dynamic Model and Vibration Power Flow of a Rigid-Flexible Coupling and Harmonic-Disturbance Exciting System for Flexible Robotic Manipulator with Elastic Joints
}

\author{
Yufei Liu, Wei Li, Yuqiao Wang, Xuefeng Yang, and Jinyong Ju \\ School of Mechatronic Engineering, China University of Mining and Technology, Xuzhou 221116, China \\ Correspondence should be addressed to Wei Li; weilicumt@yahoo.com
}

Received 21 July 2015; Revised 1 November 2015; Accepted 9 November 2015

Academic Editor: Alba Sofi

Copyright (C) 2015 Yufei Liu et al. This is an open access article distributed under the Creative Commons Attribution License, which permits unrestricted use, distribution, and reproduction in any medium, provided the original work is properly cited.

\begin{abstract}
This paper investigates the dynamic of a flexible robotic manipulator (FRM) which consists of rigid driving base, flexible links, and flexible joints. With considering the motion fluctuations caused by the coupling effect, such as the motor parameters and mechanism inertias, as harmonic disturbances, the system investigated in this paper remains a parametrically excited system. An elastic restraint model of the FRM with elastic joints (FRMEJ) is proposed, which considers the elastic properties of the connecting joints between the flexible arm and the driving base, as well as the harmonic disturbances aroused by the electromechanical coupling effect. As a consequence, the FRMEJ accordingly remains a flexible multibody system which conveys the effects of rigid-flexible couple and electromechanical couple. The Lagrangian function and Hamilton's principle are used to establish the dynamic model of the FRMEJ. Based on the dynamic model proposed, the vibration power flow is introduced to show the vibration energy distribution. Numerical simulations are conducted to investigate the effect of the joint elasticities and the disturbance excitations, and the influences of the structure parameters and motion parameters on the vibration power flow are studied. The results obtained in this paper contribute to the structure design, motion optimization, and vibration control of FRMs.
\end{abstract}

\section{Introduction}

Flexible robotic manipulators (FRMs) which consist of driving base, flexible links, and flexible joints are typical electromechanical coupling systems and have been extensively used in scientific fields, such as robotic fields and aerospace fields, which can satisfy the demands of high productivity, high speed, and lower energy consumption thanks to their lightweight advantage [1-3]. The flexible joints in the FRM remain as two types; the first type of joints is that between the flexible links, and the other type is the connecting joints between the flexible links and the driving base, such as bolting joints and welding joints. Because of lower damp and stiffness, the flexible link will exhibit elastic deformations and residual vibrations during the task executions [4, 5]. The dynamic modeling of the FRM, which is essential to investigate and control these undesirable elastic deformations and residual vibrations, generally regards the driving base as a rigid body. In this case, the elastic deformations and residual vibrations in the flexible links will be coupled with the rigidbody motion of the driving base via the flexible joints [6]; as a result, the FRM conveys rigid-flexible couple and remains a flexible multibody system. Some researchers have proposed the dynamic models of flexible multibody systems with multiple links and joints [5-8]. In their investigations, the flexible links are modeled as flexible beams and the flexible joints are characterized as tension springs and torsional springs, and the results indicate that the flexible links and flexible joints have significant influences on the dynamic behaviors of the systems. However, the flexible joints investigated in these existing studies mainly focus on the joints between the flexible links and few surveys have studied the connecting joints between the flexible links and the driving base, as the connecting joints are generally regarded as absolutely rigid. Actually, the connecting joints have certain elasticities and the ideal rigid restraint assumed is difficult to achieve.

As a rigid-flexible coupling system, the rigid-body motion of the driving base accordingly influences the 
dynamic behaviors of the flexible links, and this influence will be enhanced by the elasticities of the connecting joints $[6,9]$. Thus, to establish an accurate dynamic model for investigating the vibration characteristics of the flexible links, the coupling effect of the driving base and the elasticity properties of the connecting joints should be fully considered. In the existing literatures, the elasticity properties of the connecting joints are ignored and the coupling effect of the driving base is neglected by regarding the velocities and accelerations of the driving base to be constant without any fluctuations [10-13]. Actually, due to the coupling effects of motor parameters and mechanism inertias, the velocities and accelerations of the driving base will exhibit certain fluctuations [3, 14-17]. If we describe the motion fluctuations as harmonic disturbances, the system becomes a parametrically excited system [18]. By reviewing current studies, numerous researches about the parametrically excited systems can be seen; however, these researches mainly focus on the moving beams and plates. Feng and $\mathrm{Hu}$ [19] and Chen et al. [20-22] intensively studied the nonlinear vibrations and stabilities of axial moving beams with considering velocity disturbances. Zhang et al. [23, 24] investigated the principal parametric and internal resonances of flexible plates. Pratiher and Dwivedy [25] studied the vibrations of a lateral moving FRM which suffers harmonic excitations; however, it can be seen in their investigations that the excitations considered in their researches do not contain the actual motions of the driving base, such as the motion accelerations and the motion velocities, and that the connecting joints between the flexible links and the driving base are regarded as absolutely rigid with an ideal fixed restraint which ignores the joint elasticities.

Different with current literatures, this paper fully considers the joint elasticities and the harmonic disturbances; consequently, the FRM with elastic joints (FRMEJ) remains a multicoupling system which contains rigid-flexible couple and electromechanical couple. Due to these strong coupling effects, the FRMEJ will present complex dynamic behaviors which have not been reported in these fields. The main objective of this paper is to establish the coupling dynamic model and investigate the vibration characteristic of this multicoupling system. To establish the dynamic model of the FRMEJ, Hamilton's principle is introduced. Based on the dynamic model proposed, this paper introduces the concept of vibration power flow, which can reflect the vibration intensity and transport characteristic and already has been applied in some applications, such as beam and plate structures [26-30], vibration isolation systems [31-33], and railway $[34,35]$, to study the vibration characteristic of the FRMEJ. Based on the response characteristics of the vibration power flow, the influences of the joint elasticities and disturbance excitations, as well as the coupling effect between joint elasticities and disturbance excitations, are investigated; specifically the actual motions of the driving base are simultaneously considered during these analyses. The remainder of this paper is organized as follows. Section 2 establishes the coupling dynamic model of the FRMEJ system. Then, vibration power flow characteristics are shown in Section 3. Section 4 conducts the numerical simulations and

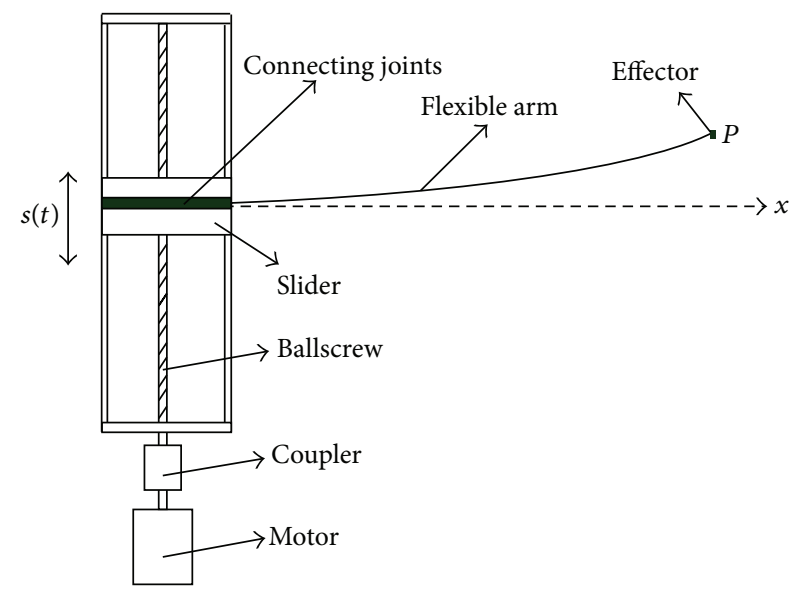

FIGURE 1: Schematic diagram of the motor driven FRM.

discusses the results. Finally, the paper is concluded with a brief summary.

\section{Coupling Dynamic Model of the FRMEJ}

Figure 1 shows a motor driven single-link FRM. The flexible arm together with the effector on its tip, which is denoted as $P$ in the figure, is clamped on the slider of the ballscrew transmission system via connecting joints. The operating tasks can be executed by the effector under the driving of the motor. In order to investigate the elastic deformations and the residual vibrations of the flexible arm, which are easily aroused during the executions because of its lower damp and stiffness properties, the dynamic model should be firstly established. During dynamic modeling, it is assumed that the flexible arm satisfies the Bernoulli-Euler beam assumption and the motion of the FRM is in the horizontal $x-y$ plane.

2.1. Motion Disturbances Equation. As indicated above, the motion characteristic of the driving base accordingly influences the dynamic behaviors of the flexible arm through the connecting joints. Thus, to investigate the vibration characteristics of the flexible arm, the motion characteristic of the driving base is firstly studied in this section.

Figure 2 shows the vibration accelerations of the driving motor under different subdivision multiples, which shows that the accelerations are not constant and exhibits certain disturbances. Through expanding the vibration signals into the form of Fourier series and using sinusoidal functions to describe the disturbances, the motion displacements of the driving base can be written as

$$
s(t)=a t^{2}+\gamma \sin \varepsilon t ;
$$

here $a$ denotes the average motion acceleration of the driving base and $\gamma$ and $\varepsilon$ denote the amplitudes and frequencies of the disturbances, respectively. In this case, the FRMEJ remains a parametrically excited system and the vibration displacements can be written as

$$
y(x, t)=s(t)+w(x, t)
$$



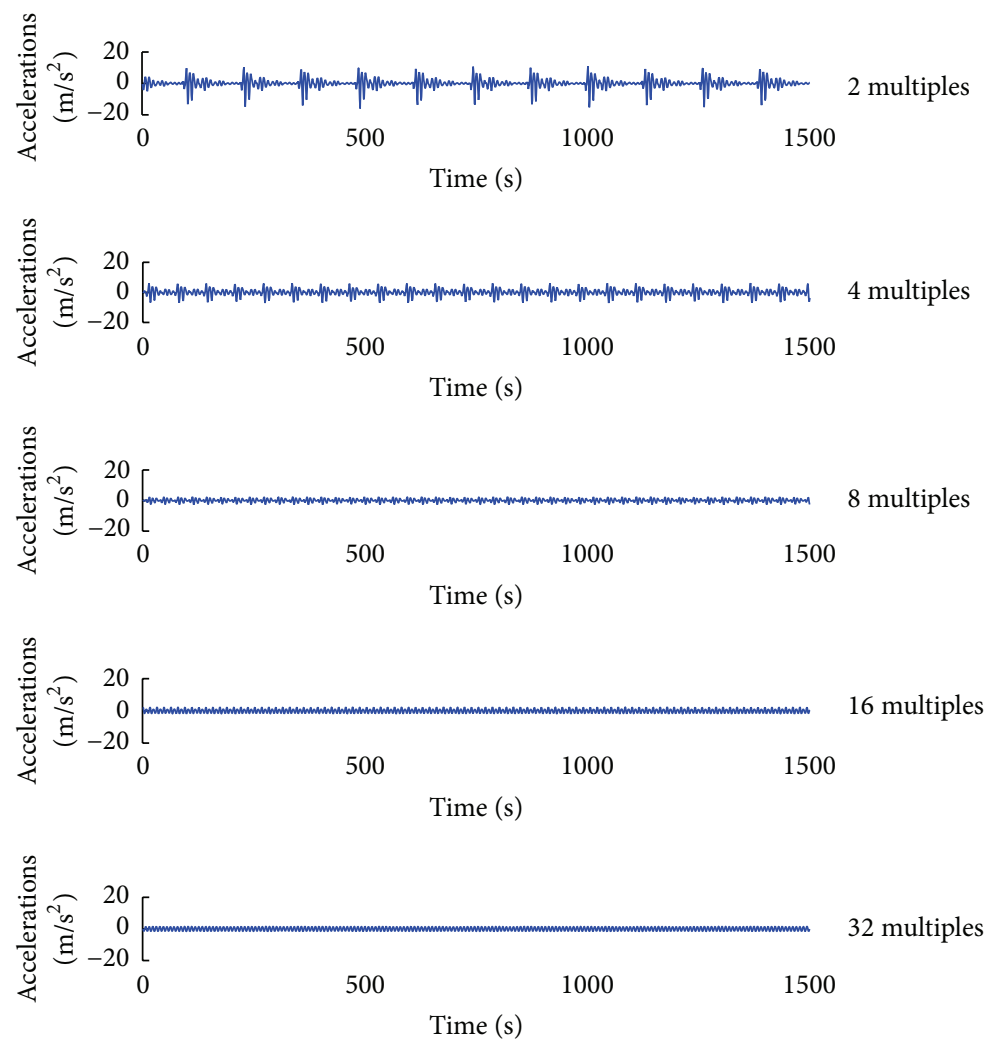

FIGURE 2: Vibration accelerations of the driving motor with different subdivision multiples.

here $w(x, t)$ denotes transverse vibration displacement of the flexible arm; according to the assumed modes method [10, 36], it yields

$$
w(x, t)=\sum_{i=1}^{n \rightarrow \infty} \phi_{i}(x) q_{i}(t),
$$

where $q_{i}(t)$ denotes the $i$ th generalized coordinate and $\phi_{i}(x)$ denotes the $i$ th mode shape, which can be given as [36]

$$
\begin{aligned}
\phi_{i}(x)= & \xi_{1} \cos \omega_{i} x+\xi_{2} \sin \omega_{i} x+\xi_{3} \cosh \omega_{i} x \\
& +\xi_{4} \sinh \omega_{i} x,
\end{aligned}
$$

where $\omega_{i}$ is the $i$ th natural frequency of the flexible arm and $\xi_{1}, \xi_{2}, \xi_{3}$, and $\xi_{4}$ are coefficients determined by boundary conditions.

The kinetic energy of the FRMEJ is

$$
\begin{aligned}
T & =\frac{1}{2} m_{s}\left(\frac{d s}{d t}\right)^{2}+\frac{1}{2} \int_{0}^{L} \rho A\left(\frac{\partial y}{\partial t}\right)^{2} d x \\
& =\frac{1}{2} m_{s}\left(\frac{d s}{d t}\right)^{2}+\frac{1}{2} \int_{0}^{L} \rho A\left(\frac{d s}{d t}+\frac{\partial w}{\partial t}\right)^{2} d x
\end{aligned}
$$

here, the first part of the equation denotes kinetic energy of the driving base and the second part denotes kinetic energy of the flexible arm; $m_{s}$ is the mass of the slider; $L, \rho$, and $A$ are the length, mass density, and cross-sectional area of the flexible arm, respectively.

The potential energy of the FRMEJ mainly considers the elastic potential energy of the flexible arm and can be written as

$$
V=\frac{1}{2} \int_{0}^{L} E I\left(\frac{\partial^{2} w}{\partial x^{2}}\right)^{2} d x
$$

where $E$ is Young's modulus and $I$ is the cross-sectional moment of inertia about the neural axis and can be expressed as $I=b h^{3} / 12$, where $b$ and $h$ are the width and thickness of the flexible arm, respectively.

To establish the dynamic model, substitute (4) and (5) into the Lagrangian function introduced as follows:

$$
U=T-V .
$$

According to Hamilton's principle [37], (7) satisfies

$$
\delta \int_{t_{0}}^{t_{1}} U d t=\int_{t_{0}}^{t_{1}} \delta(T-V) d t=0
$$


here

$$
\begin{aligned}
& \delta \int_{t_{0}}^{t_{1}} T d t \\
& =\int_{t_{0}}^{t_{1}} \delta\left[\frac{1}{2} m_{s}\left(\frac{d s}{d t}\right)^{2}+\frac{1}{2} \int_{0}^{L} \rho A\left(\frac{d s}{d t}+\frac{\partial w}{\partial t}\right)^{2} d x\right] d t \\
& =\int_{t_{0}}^{t_{1}} \int_{0}^{L} \rho A\left(\frac{d s}{d t}+\frac{\partial w}{\partial t}\right) \delta \frac{\partial w}{\partial t} d x d t \\
& =\int_{t_{0}}^{t_{1}} \int_{0}^{L} \rho A\left(\frac{d s}{d t}+\frac{\partial w}{\partial t}\right) d x d(\delta w) \\
& =-\int_{t_{0}}^{t_{1}} \int_{0}^{L} \rho A\left(\frac{d^{2} s}{d t^{2}}+\frac{\partial^{2} w}{\partial t^{2}}\right) d x d t \delta w, \\
& \delta \int_{t_{0}}^{t_{1}} V d t=\int_{t_{0}}^{t_{1}} \delta\left[\frac{1}{2} \int_{0}^{L} E I\left(\frac{\partial^{2} w}{\partial x^{2}}\right)^{2} d x\right] d t \\
& =\int_{t_{0}}^{t_{1}} \int_{0}^{L} E I \frac{\left(\partial^{2} w / \partial x^{2}\right)\left(\partial^{3} w / \partial x^{3}\right)}{\partial w / \partial x} d x d t \delta w . \\
& \delta \int_{t_{0}}^{t_{1}} U d t=-\int_{t_{0}}^{t_{1}} \int_{0}^{L} \rho A\left(\frac{d^{2} s}{d t^{2}}+\frac{\partial^{2} w}{\partial t^{2}}\right) d x d t \delta w \\
& -\int_{t_{0}}^{t_{1}} \int_{0}^{L} E I \frac{\left(\partial^{2} w / \partial x^{2}\right)\left(\partial^{3} w / \partial x^{3}\right)}{\partial w / \partial x} d x d t \delta w \\
& =-\int_{t_{0}}^{t_{1}} \int_{0}^{L}\left[\rho A\left(\frac{d^{2} s}{d t^{2}}+\frac{\partial^{2} w}{\partial t^{2}}\right)\right. \\
& \left.+E I \frac{\left(\partial^{2} w / \partial x^{2}\right)\left(\partial^{3} w / \partial x^{3}\right)}{\partial w / \partial x}\right] d x d t \delta w \\
& =0 \text {. }
\end{aligned}
$$

From (10), it can be obtained that

$$
\rho A\left(\frac{d^{2} s}{d t^{2}}+\frac{\partial^{2} w}{\partial t^{2}}\right)+E I \frac{\left(\partial^{2} w / \partial x^{2}\right)\left(\partial^{3} w / \partial x^{3}\right)}{\partial w / \partial x}=0 .
$$

By combining (3), (11) can be further written as

$$
\rho A \ddot{s}+\rho A \phi_{i} \ddot{q}_{i}+E I \omega_{i}^{4} \phi_{i} q_{i}=0, \quad i=1,2, \ldots, n ;
$$

here $(\cdot)$ denotes the time derivative.

Through multiplying (12) with $\phi_{j}$ and integrating the equation along length direction, result can be obtained that

$$
\begin{aligned}
\int_{0}^{L} \phi_{j}\left(\rho A \ddot{s}+\rho A \phi_{i} \ddot{q}_{i}+E I \omega_{i}^{4} \phi_{i} q_{i}\right) d x= & , \\
& j=1,2, \ldots, n .
\end{aligned}
$$

According to the orthogonality of mode shapes [11, 38], the vibration displacement equation of the FRMEJ can be obtained as

$$
\ddot{q}_{i}+\omega_{i}^{2} q_{i}=-\frac{m_{i}}{\rho A} \ddot{s}
$$

where

$$
m_{i}=\int_{0}^{L} \rho A \phi_{i} d x
$$

According to the Duhamel integral [36], the vibration responses of the FRMEJ can be expressed as

$$
\begin{aligned}
q_{i}(t)= & b_{1 i} \cos \omega_{i} t+b_{2 i} \sin \omega_{i} t \\
& -\frac{1}{\omega_{i}} \int_{0}^{t} \frac{m_{i}}{\rho A} \ddot{s}(\tau) \sin \omega_{i}(t-\tau) d \tau
\end{aligned}
$$

here $b_{1 i}$ and $b_{2 i}$ are coefficients determined by the initial conditions and can be expressed as $[11,38]$

$$
\begin{aligned}
& b_{1 i}=\frac{\rho A}{M_{i}} \int_{0}^{L} w(x, 0) \phi_{i} d x, \\
& b_{2 i}=\frac{\rho A}{M_{i} \omega_{i}} \int_{0}^{L} \frac{\partial w(x, 0)}{\partial t} \phi_{i} d x,
\end{aligned}
$$

where $M_{i}$ denotes the generalized mass of the $i$ th mode and can be defined as

$$
M_{i}=\int_{0}^{L} \rho A \phi_{i}^{2} d x
$$

By combining (1) and (16), it can be obtained that

$$
\begin{aligned}
q_{i}(t)= & b_{1 i} \cos \omega_{i} t+b_{2 i} \sin \omega_{i} t \\
& +\frac{m_{i}}{\rho A} \frac{\gamma \varepsilon^{2}\left(\varepsilon \sin \omega_{i} t-\omega_{i} \sin \varepsilon t\right)}{\omega_{i}\left(\varepsilon^{2}-\omega_{i}^{2}\right)} \\
& -\frac{2 a m_{i}}{\rho A \omega_{i}^{2}}\left(1-\cos \omega_{i} t\right) .
\end{aligned}
$$

2.2. Elastic Restraint Model. From (19), it can be obtained that the vibration responses of the FRMEJ are related to the motion disturbances. According to (3), to investigate the vibration responses of the FRMEJ, the mode shapes should also be determined. Considering the fact that the connecting joints between the flexible arm and the driving base, such as bolted joints and welding joints, actually exhibit certain elasticities, as a consequence, the restraints of the connecting joints are not ideally fixed which is difficult to achieve in fact. Thus, an accurate restraint model should be proposed to describe the real restraints of the joints. To describe the joint elasticities, we model the joints as tension spring and torsional spring, with restraint rigidities $k$ and $k_{t}$, respectively. In this situation, an elastic restraint model is established as shown in Figure 3. It can be seen that, under the harmonic disturbances, the FRMEJ remains a multicoupling system which conveys rigid-flexible couple and parametric excitations. 


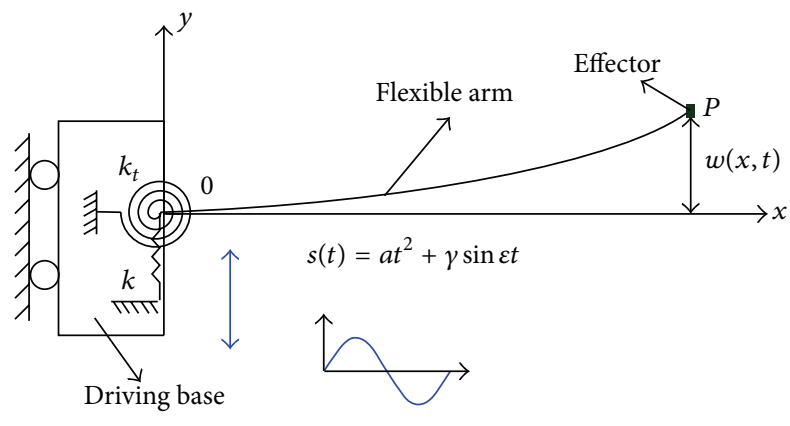

FIGURE 3: Elastic restraint model of the FRMEJ.

Based on the proposed elastic restraint model and according to the balances of forces and moments [39], it can be obtained that

$$
\begin{array}{r}
k w+E I \frac{\partial^{3} w}{\partial x^{3}}=0 \\
k_{t} \frac{\partial w}{\partial x}+E I \frac{\partial^{2} w}{\partial x^{2}}=0
\end{array}
$$

here, $k w$ and $k_{t}(\partial w / \partial x)$ denote the force and moment of the tension spring and torsional spring; $E I\left(\partial^{3} w / \partial x^{3}\right)$ and $E I\left(\partial^{2} w / \partial x^{2}\right)$ denote the shear force and moment generated in the flexible arm, respectively.

By assigning $x=0$ in (20), the boundary condition of the fixed end can be obtained as

$$
\begin{aligned}
k w(0, t)+E I \frac{\partial^{3} w(0, t)}{\partial x^{3}} & =0 \\
k_{t} \frac{\partial w(0, t)}{\partial x}+E I \frac{\partial^{2} w(0, t)}{\partial x^{2}} & =0 .
\end{aligned}
$$

Because the shear force and moment of the free end are zero, assigning $x=L$ in (20) can obtain the boundary condition of free end:

$$
\begin{aligned}
& \frac{\partial^{3} w(L, t)}{\partial x^{3}}=0 \\
& \frac{\partial^{2} w(L, t)}{\partial x^{2}}=0 .
\end{aligned}
$$

According to (3), (21) and (22) can be further written as

$$
\begin{aligned}
k \phi(0, t)+E I \frac{\partial^{3} \phi(0, t)}{\partial x^{3}} & =0 \\
k_{t} \frac{\partial \phi(0, t)}{\partial x}+E I \frac{\partial^{2} \phi(0, t)}{\partial x^{2}} & =0 \\
\frac{\partial^{3} \phi(L, t)}{\partial x^{3}} & =0 \\
\frac{\partial^{2} \phi(L, t)}{\partial x^{2}} & =0 .
\end{aligned}
$$

Combining (4) and (23) yields

$$
\begin{aligned}
\left(\xi_{1}+\xi_{3}\right) k-\left(\xi_{2}-\xi_{4}\right) E I \omega^{3} & =0, \\
\left(\xi_{2}+\xi_{4}\right) k_{t} \omega-\left(\xi_{1}-\xi_{3}\right) E I \omega^{2} & =0 .
\end{aligned}
$$

Similarly, combining (4) and (24) yields

$$
\begin{aligned}
\xi_{1} \sin \omega L-\xi_{2} \cos \omega L+\xi_{3} \sinh \omega L+\xi_{4} \cosh \omega L & =0, \\
-\xi_{1} \cos \omega L-\xi_{2} \sin \omega L+\xi_{3} \cosh \omega L+\xi_{4} \sinh \omega L & =0 .
\end{aligned}
$$

With the purpose of simplifying the following analysis, (25) and (26) are expressed as

$$
\begin{gathered}
{\left[\begin{array}{cc}
k & -\lambda \omega \\
\lambda & k_{t} \omega
\end{array}\right]\left[\begin{array}{l}
\xi_{1} \\
\xi_{2}
\end{array}\right]+\left[\begin{array}{ll}
k & \lambda \omega \\
\lambda & k_{t} \omega
\end{array}\right]\left[\begin{array}{l}
\xi_{3} \\
\xi_{4}
\end{array}\right]=0,} \\
{\left[\begin{array}{cc}
\sin \omega L & -\cos \omega L \\
-\cos \omega L & -\sin \omega L
\end{array}\right]\left[\begin{array}{l}
\xi_{1} \\
\xi_{2}
\end{array}\right]} \\
+\left[\begin{array}{ll}
\sinh \omega L & \cosh \omega L \\
\cosh \omega L & \sinh \omega L
\end{array}\right]\left[\begin{array}{l}
\xi_{3} \\
\xi_{4}
\end{array}\right]=0
\end{gathered}
$$

where

$$
\lambda=E I \omega^{2} \text {. }
$$

From (27), it can be obtained that

$$
\begin{aligned}
& \xi_{1}=-\left(1+\frac{2 \lambda^{2}}{k k_{t}-\lambda^{2}}\right) \xi_{3}-\frac{2 k_{t} \lambda \omega}{k k_{t}-\lambda^{2}} \xi_{4}, \\
& \xi_{2}=-\frac{2 k \lambda}{\left(k k_{t}-\lambda^{2}\right) \omega} \xi_{3}-\left(1+\frac{2 \lambda^{2}}{k k_{t}-\lambda^{2}}\right) \xi_{4} .
\end{aligned}
$$

Combining (27) and (28) obtains

$$
\left[\begin{array}{ll}
\Delta_{11} & \Delta_{12} \\
\Delta_{21} & \Delta_{22}
\end{array}\right]\left[\begin{array}{l}
\xi_{3} \\
\xi_{4}
\end{array}\right]=0
$$

where

$$
\begin{aligned}
\Delta_{11}= & k(\sinh \omega L-\sin \omega L)-\lambda \omega(\cosh \omega L-\cos \omega L), \\
\Delta_{12}= & k(\cosh \omega L+\cos \omega L)-\lambda \omega(\sinh \omega L-\sin \omega L), \\
\Delta_{21}= & -\lambda(\sinh \omega L+\sin \omega L) \\
& +k_{t} \omega(\cosh \omega L+\cos \omega L), \\
\Delta_{22}= & -\lambda(\cosh \omega L-\cos \omega L) \\
& +k_{t} \omega(\sinh \omega L+\sin \omega L) .
\end{aligned}
$$

Through substituting (30) into (4), the mode shape of the FRMEJ can be obtained as

$$
\begin{array}{r}
\phi(x)=\left[(\sin \omega x-\sinh \omega x)+\frac{2 k_{t} \lambda \omega}{k k_{t}-\lambda^{2}} \cos \omega x\right. \\
\left.+\frac{2 \lambda^{2}}{k k_{t}-\lambda^{2}} \sin \omega x\right]+\zeta[(\cos \omega x-\cosh \omega x) \\
\left.+\frac{2 \lambda^{2}}{k k_{t}-\lambda^{2}} \cos \omega x+\frac{2 k \lambda}{\left(k k_{t}-\lambda^{2}\right) \omega} \sin \omega x\right],
\end{array}
$$


where

$$
\begin{gathered}
\zeta=\frac{\xi_{3}}{\xi_{4}}=-\frac{\Delta_{22}}{\Delta_{21}} \\
=-\frac{(\sinh \omega L+\sin \omega L)-\left(\lambda / k_{t} \omega\right)(\cosh \omega L-\cos \omega L)}{(\cosh \omega L+\cos \omega L)-\left(\lambda / k_{t} \omega\right)(\sinh \omega L+\sin \omega L)} . \\
\text { Assigning } k \rightarrow \infty \text { and } k_{t} \rightarrow \infty \text { in }(33) \text { yields } \\
\phi(x)=\sin \omega x-\sinh \omega x \\
\quad-\frac{\sin \omega L+\sinh \omega L}{\cos \omega L+\cosh \omega L}(\cos \omega x-\cosh \omega x) .
\end{gathered}
$$

Equation (35) describes the mode shape of the situation that the connecting joint has a fixed restraint [36]; as the assumed condition $k \rightarrow \infty, k_{t} \rightarrow \infty$ is difficult to achieve in fact, we can realize that the fixed restraint is an ideal status which cannot reflect the real restraint and will cause an error, especially for the precision system. It can be seen that the proposed elastic model provides a description of the elastic restraint and is also suitable for other restraint cases.

\section{Vibration Power Flow of the FRMEJ}

Equations (19) and (33) can provide a description of the influences of the motion disturbances and joint elasticities on the mode and vibration responses. By combining (2), (19), and (33), the vibration displacements of the FRMEJ can be subsequently obtained as

$$
\begin{gathered}
w(x, t)=\sum_{i=1}^{n \rightarrow \infty} \phi_{i}(x)\left[b_{1 i} \cos \omega_{i} t+b_{2 i} \sin \omega_{i} t\right. \\
+\frac{m_{i}}{\rho A} \frac{\gamma \varepsilon^{2}\left(\varepsilon \sin \omega_{i} t-\omega_{i} \sin \varepsilon t\right)}{\omega_{i}\left(\varepsilon^{2}-\omega_{i}^{2}\right)} \\
\left.-\frac{2 a m_{i}}{\rho A \omega_{i}^{2}}\left(1-\cos \omega_{i} t\right)\right] .
\end{gathered}
$$

In order to investigate the characteristic of the vibration energy distribution of the FRMEJ and study the influence of the motion disturbances, the vibration power flow is introduced in this section and can be written as [29-31, 40, 41]

$$
P(x)=\frac{E I \omega_{i}}{2 \pi} \int_{0}^{2 \pi / \omega_{i}}\left(\frac{\partial^{3} w}{\partial x^{3}} \frac{\partial w}{\partial t}+\frac{\partial^{2} w}{\partial x^{2}} \frac{\partial^{2} w}{\partial x \partial t}\right) d t
$$

where

$$
\begin{aligned}
\frac{\partial^{3} w}{\partial x^{3}} & =\sum_{i=1}^{n \rightarrow \infty} \frac{d^{3} \phi_{i}(x)}{d x^{3}} q_{i}(t), \\
\frac{\partial^{2} w}{\partial x^{2}} & =\sum_{i=1}^{n \rightarrow \infty} \frac{d^{2} \phi_{i}(x)}{d x^{2}} q_{i}(t), \\
\frac{\partial^{2} w}{\partial x \partial t} & =\sum_{i=1}^{n \rightarrow \infty} \frac{d \phi_{i}(x)}{d x} \dot{q}_{i}(t), \\
\frac{\partial w}{\partial t} & =\sum_{i=1}^{n \rightarrow \infty} \phi_{i}(x) \dot{q}_{i}(t) .
\end{aligned}
$$

Substituting (38)-(41) into (37) yields

$$
P(x)=\frac{E I \omega_{i}}{2 \pi}\left(\phi_{i}^{\prime \prime \prime} \phi_{i}+\phi_{i}^{\prime \prime} \phi_{i}^{\prime}\right) \int_{0}^{2 \pi / \omega_{i}} \dot{q}_{i} q_{i} d t .
$$

Combining (19) obtains

$$
\begin{aligned}
& \int_{0}^{2 \pi / \omega_{i}} \dot{q}_{i} q_{i} d t=\frac{1}{2} \frac{m_{i}}{\rho A} \\
& . \frac{\gamma \varepsilon^{2} \sin \left(2 \pi \varepsilon / \omega_{i}\right)}{\varepsilon^{2}-\omega_{i}^{2}}\left(\frac{m_{i}}{\rho A} \frac{\gamma \varepsilon^{2} \sin ^{2}\left(2 \pi \varepsilon / \omega_{i}\right)}{\varepsilon^{2}-\omega_{i}^{2}}-2 b_{1 i}\right) .
\end{aligned}
$$

With the substitution of (43) into (42), the vibration power flow of the FRMEJ can be obtained as

$$
\begin{aligned}
& P(x)=\frac{E I}{4 \pi \rho A} \sum_{i=1}^{n \rightarrow \infty} m_{i} \omega_{i} \\
& . \frac{\gamma \varepsilon^{2} \sin \left(2 \pi \varepsilon / \omega_{i}\right)}{\varepsilon^{2}-\omega_{i}^{2}}\left(\frac{m_{i}}{\rho A} \frac{\gamma \varepsilon^{2} \sin \left(2 \pi \varepsilon / \omega_{i}\right)}{\varepsilon^{2}-\omega_{i}^{2}}-2 b_{1 i}\right) \\
& \cdot\left(\phi_{i}^{\prime \prime \prime} \phi_{i}+\phi_{i}^{\prime \prime} \phi_{i}^{\prime}\right) .
\end{aligned}
$$

Equation (44) presents a description that the vibration power flow is related to the motion disturbances; moreover, the initial condition also has an influence on the vibration power flow, which will be further illustrated in Section 4. On the other hand, we can see that the vibration power flow is a continuum, which can clearly show the vibration energy distributions of the system and is meaningful for further vibration control, for example, the location optimizations of the sensors and actuators.

\section{Results and Discussions}

In this section, to investigate the vibration power flow characteristic of the FRMEJ, numerical simulations are conducted. During the analysis, the flexible arm is characterized as a flexible beam to describe its lower damp and stiffness feature, with properties as follows: $L=575 \mathrm{~mm}, b=28 \mathrm{~mm}, h=$ $1 \mathrm{~mm}, E=197 \mathrm{GPa}, \rho=7850 \mathrm{~kg} / \mathrm{m}^{3}$, and $\mu=0.26$.

The vibration power flow responses of the FRMEJ with different disturbance amplitudes $(\gamma)$ and disturbance frequencies $(\varepsilon)$ are shown in Figures 4-6. According to Figures 4 and 5, it can be obtained that the disturbance amplitudes $(\gamma)$ and disturbance frequencies $(\varepsilon)$ have considerable influences on the vibration power flow. Specifically, the response amplitudes increase with the disturbance amplitudes $(\gamma)$ and disturbance frequencies $(\varepsilon)$.

Figures 4 and 5 indicate that the disturbance amplitudes $(\gamma)$ and disturbance frequencies $(\varepsilon)$ all have influence on the vibration power flow. In order to compare the influences of the disturbance amplitudes $(\gamma)$ and disturbance frequencies $(\varepsilon)$, Figure 6 presents the vibration power flow of the FRMEJ with assigning $\gamma_{1}=1 \mathrm{~mm}, \varepsilon_{1}=15 \mathrm{~Hz}$ and $\gamma_{2}=3 \mathrm{~mm}$, $\varepsilon_{2}=5 \mathrm{~Hz}$, which conveys the relationship that $\varepsilon_{1}$ is three times $\varepsilon_{2}$ while $\gamma_{2}$ is three times $\gamma_{1}$. From Figure 6 , it can be seen that the vibration power flow amplitude with $\gamma_{1}=1 \mathrm{~mm}$ 


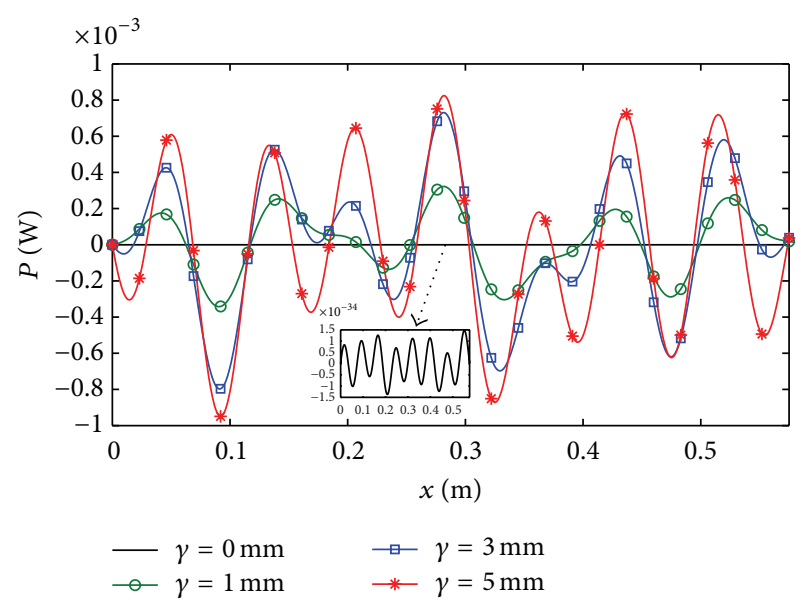

FIgURE 4: Vibration power flow of the FRMEJ with different disturbance amplitudes.

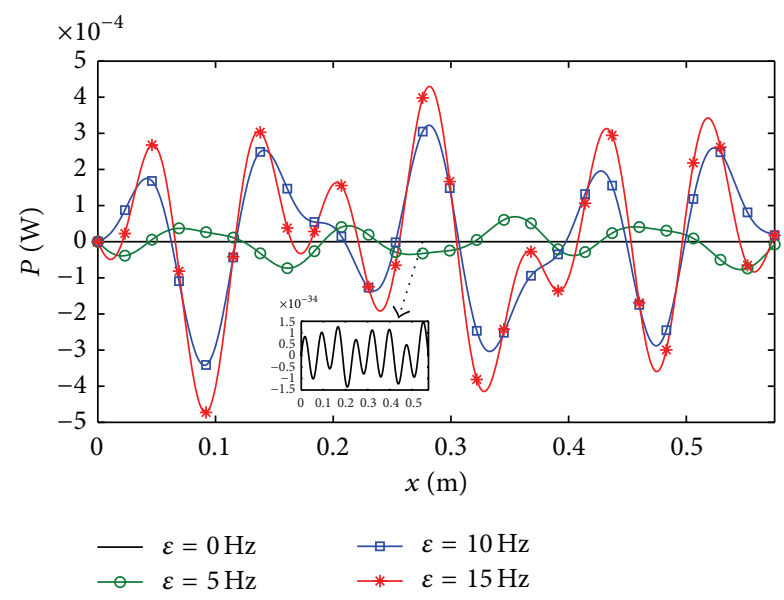

FIGURE 5: Vibration power flow of the FRMEJ with different disturbance frequencies.

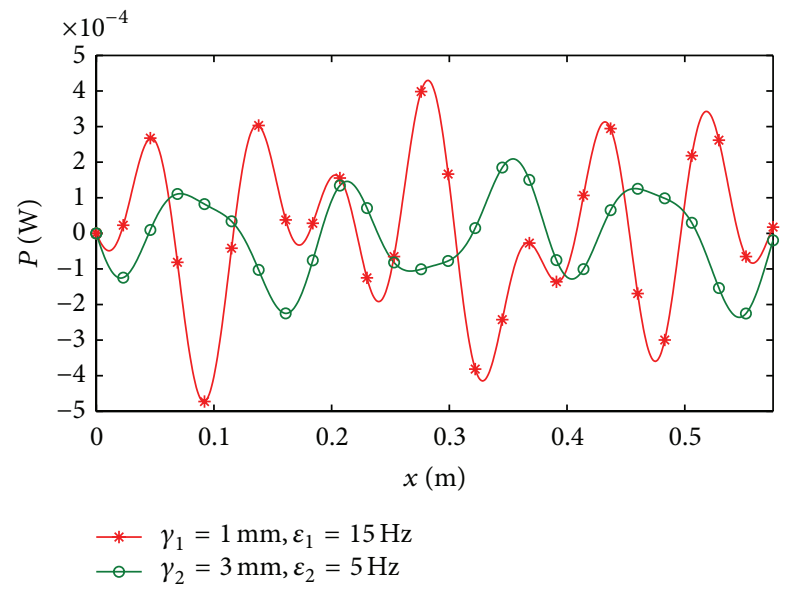

FIGURE 6: Vibration power flow of the FRMEJ with different disturbance amplitudes and frequencies.

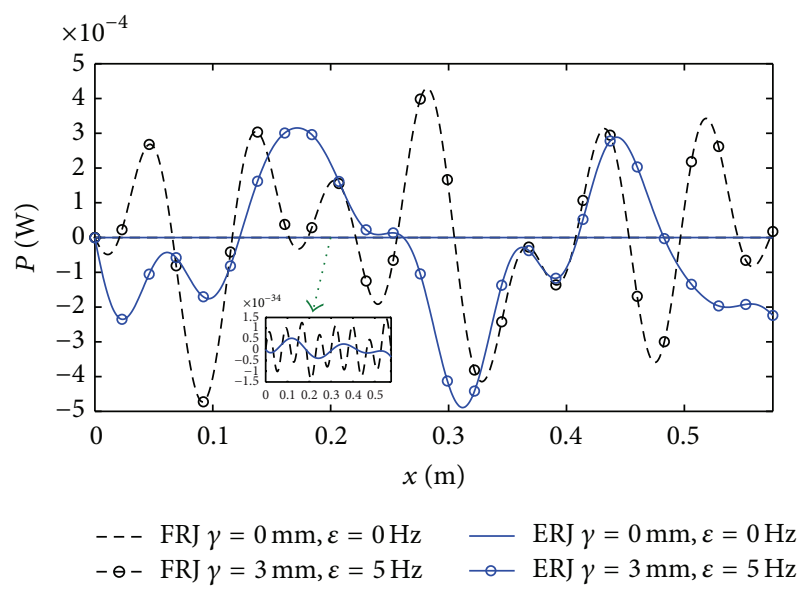

FIGURE 7: Vibration power flow of the FRM with FRJ and ERJ under disturbance excitations.

and $\varepsilon_{1}=15 \mathrm{~Hz}$ is larger than that of $\gamma_{2}=3 \mathrm{~mm}$ and $\varepsilon_{2}=$ $5 \mathrm{~Hz}$; this reveals that the influence of disturbance frequencies $(\varepsilon)$ on the vibration power flow is more pronounced than disturbance amplitudes $(\gamma)$; and the motion disturbances affect the vibration characteristic, primarily through the disturbance frequencies $(\varepsilon)$.

As indicated above, the restraints of the connecting joints are regarded as fixed in ideal status, and actually the joints exhibit certain elasticities. To further investigate the influence of the motion disturbances, Figure 7 shows the vibration power flow of the FRM with fixed restraint joints (FRJ) and elastic restraint joints (ERJ). It can be obtained that if the FRM does not suffer motion disturbances, denoted as $\gamma=0 \mathrm{~mm}$ and $\varepsilon=0 \mathrm{~Hz}$, the amplitude of ERJ is smaller than that of FRJ, which reveals that the vibration power flow characteristic of the FRM with elastic restraint is different from that of fixed restraint and that the joint elasticities have suppressing effect on the vibration responses. However, when the FRM suffers motion disturbances, denoted as $\gamma=3 \mathrm{~mm}$ and $\varepsilon=5 \mathrm{~Hz}$, the amplitude of ERJ is larger than that of FRJ. On the other hand, Figure 8 further shows the vibration power flow of the FRMEJ with different restraint rigidities under the motion disturbance, in which we obtain the fact that the amplitudes decrease and gradually approach to the status of FRJ with the restraint rigidities increasing.

Moreover, as the effector is clamped on the tip of the flexible arm (as denoted in Figure 1), from Figures 7 and 8, we can see that the vibration responses of the effector with ERJ are larger than that of FRJ, and this reveals that, under the motion disturbances, the vibration responses of the effector are also enlarged, which is contrary to the demands of operating accuracy and consequently decreases the service life of the system; this further shows that the motion disturbances affect the dynamic performance and enlarge the vibration responses, especially for the FRM with ERJ, which should be suppressed in actual engineering applications.

From the comprising description of the FRM as shown in Figure 1, we can see that the flexible arm together with the effector is the main component of the operating system. 


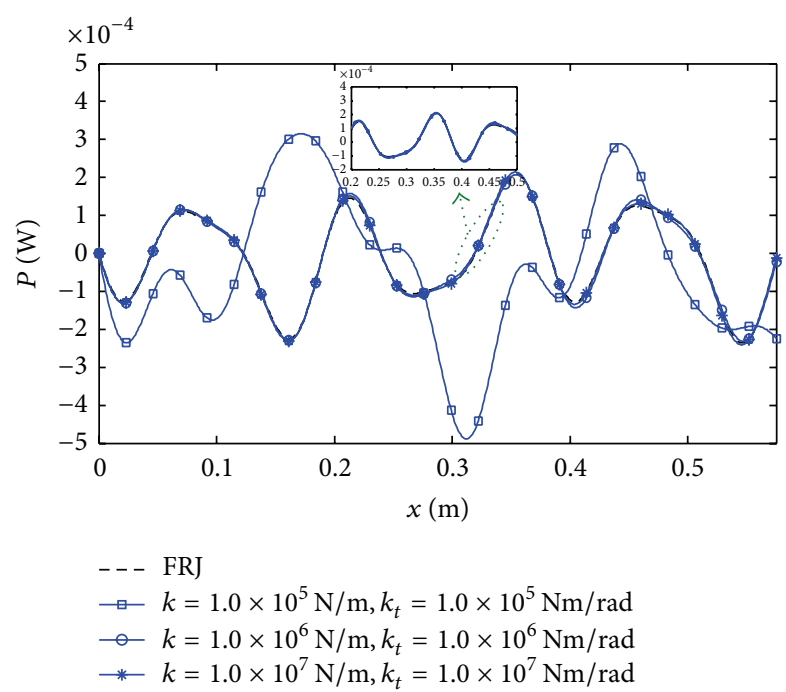

FIGURE 8: Vibration power flow of the FRMEJ with different restraint rigidities under the motion disturbance.

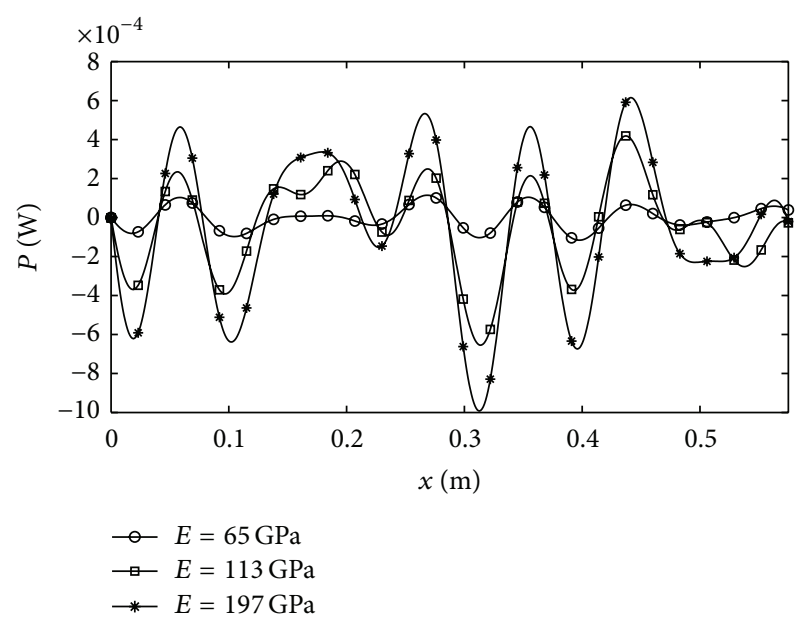

FIgURE 9: Vibration power flow of the FRMEJ with different modules under disturbance excitations.

Generally, the flexible arms of the FRMs are constructed using lightweight materials and structures which have lower Young's modulus. Figure 9 shows the vibration power flow of the FRMEJ with different Young's modulus, assigning $E=$ $65 \mathrm{Gpa}, 113 \mathrm{Gpa}$, and $197 \mathrm{Gpa}$. During the analysis, the motion disturbance is assigned $\gamma=3 \mathrm{~mm}$ and $\varepsilon=5 \mathrm{~Hz}$. From Figure 9, it can be seen that the amplitudes increase with the increasing of Young's modulus and Young's modulus has a considerable influence on the vibration power flow, which is significant for the structure designs of FRMs.

As the FRMEJ generally has actual acceleration motions, Figure 10 shows the vibration power flow of the FRMEJ with $a=0.01 \mathrm{~m} / \mathrm{s}^{2}, 0.02 \mathrm{~m} / \mathrm{s}^{2}$, and $0.03 \mathrm{~m} / \mathrm{s}^{2}$. It can be seen that the vibration power flow responses of different motion accelerations present tiny change, which indicates that the motion accelerations have no influence on the vibration power flow. On the other hand, the result further shows that

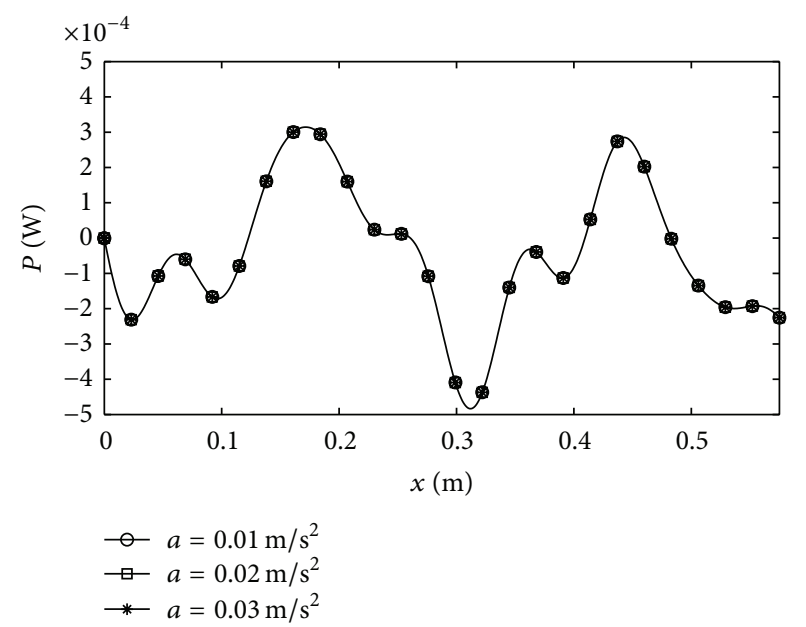

FIGURE 10: Vibration power flow of the FRMEJ with different accelerations under disturbance excitations.

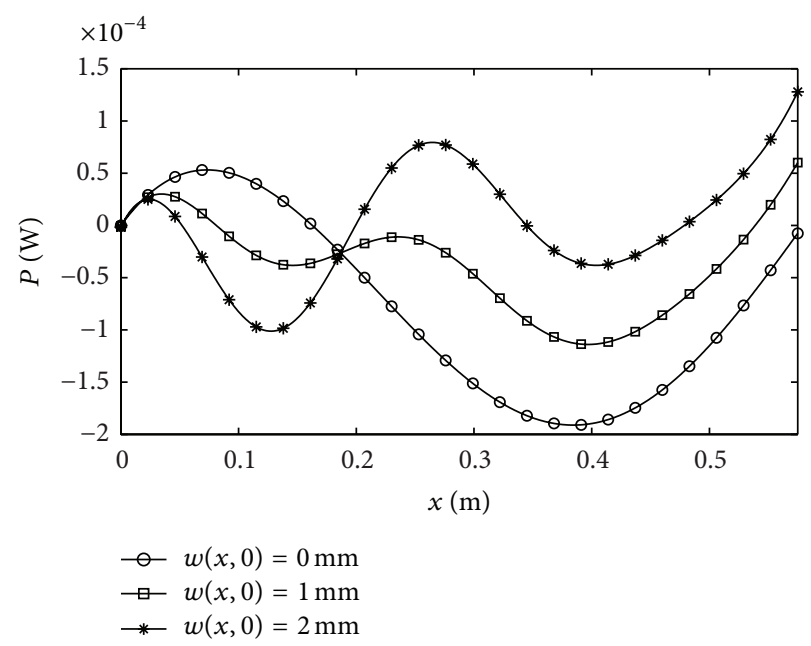

FIGURE 11: Effect of initial conditions on the vibration power flow of the FRMEJ with disturbance excitations.

the motion accelerations have no effect on the influence of the motion disturbances which is defined as $\gamma=3 \mathrm{~mm}$ and $\varepsilon=5 \mathrm{~Hz}$ during the analysis.

Moreover, (41) shows that the vibration power flow is also related to $b_{1}$ which denotes the initial conditions. In order to present a further study about this, Figure 11 presents the vibration power flow of the FRMEJ with different initial conditions. We can see that the vibration power flow characteristics vary with the initial conditions; as the initial displacement increases, the response amplitudes of the effector increase. This indicates that we can control and optimize the start-up characteristics of the driving motor, for example, the power frequencies, to obtain the desired dynamic performance of the FRM and subsequently achieve the operating accuracy of the effector. 


\section{Conclusions}

Considering the elastic properties of the connecting joints and the motion disturbances of the driving base, the flexible robotic manipulator (FRM) remains a multicoupling system conveying rigid-flexible couple and electromechanical couple. The coupling dynamic model is proposed and the vibration power flow characteristics of the FRM with elastic joints (FRMEJ) are investigated. Results indicate that, without motion disturbances, the joint elasticities present suppressing effect on the vibrations responses, which is meaningful for the structure design of the FRM, while the motion disturbances have considerable influences on the dynamic performance of the FRME. The response amplitudes of the vibration power flow increase with the disturbance amplitudes and disturbance frequencies, and moreover, the effect of the disturbance frequencies is more pronounced than the disturbance amplitudes. On the other hand, under the motion disturbances, the vibration power flow amplitudes of the FRMEJ with ERJ are larger than that of FRJ; with the restraint rigidities increasing, the amplitudes decrease and gradually approach to the status of FRJ, which shows that the motion disturbances enlarge the vibration responses of the flexible arm and the effector, especially for the FRM with ERJ, which should be suppressed in actual engineering applications. Moreover, Young's modulus and initial conditions also affect the vibration power flow characteristic of the FRMEJ. Actually, based on the results in this paper, we can achieve the expected dynamic performance of the FRMEJ through the optimizations of structure parameters design, such as Young's modulus and the restraint rigidities of the connecting joints, and the motion property, for example, the starting power frequencies. Also, based on the dynamic model proposed, the vibration control strategies can be conducted to suppress the motion disturbances of the driving base as well as the vibration responses of the flexible arm and the effector, which will be conducted in our further work. The modeling approach and results contribute to the investigations of dynamic and vibration control of multicoupling systems.

\section{Conflict of Interests}

The authors declare that there is no conflict of interests regarding the publication of this paper.

\section{Acknowledgments}

This research work is supported by the National Natural Science Foundation of China (no. 51305444), the Doctoral Fund of Ministry of Education (no. 20120095120013), the Scientific and Technological Project of Jiangsu Province (BY2014028-06), and the Project Funded by the Priority Academic Program Development of Jiangsu Higher Education Institutions (PAPD).

\section{References}

[1] S. K. Dwivedy and P. Eberhard, "Dynamic analysis of flexible manipulators, a literature review," Mechanism and Machine Theory, vol. 41, no. 7, pp. 749-777, 2006.
[2] H. N. Rahimi and M. Nazemizadeh, "Dynamic analysis and intelligent control techniques for flexible manipulators: a review," Advanced Robotics, vol. 28, no. 2, pp. 63-76, 2014.

[3] S. Bruno and K. Oussama, Springer Handbook of Robotics, Springer, London, UK, 2008.

[4] F. Xi and R. G. Fenton, "Coupling effect of a flexible link and a flexible joint," The International Journal of Robotics Research, vol. 13, no. 5, pp. 443-453, 1994.

[5] B. Subudhi and A. S. Morris, "Dynamic modelling, simulation and control of a manipulator with flexible links and joints," Robotics and Autonomous Systems, vol. 41, no. 4, pp. 257-270, 2002.

[6] W. Chen, "Dynamic modeling of multi-link flexible robotic manipulators," Computers \& Structures, vol. 79, no. 2, pp. 183195, 2001.

[7] Y. Wang and R. L. Huston, "A lumped parameter method in the nonlinear analysis of flexible multibody systems," Computers \& Structures, vol. 50, no. 3, pp. 421-432, 1994.

[8] Z. J. Qian and D. G. Zhang, "Impact dynamics of multi-link robots with link and joint flexibility," Applied Mechanics and Materials, vol. 226-228, pp. 685-692, 2012.

[9] Y. F. Liu, W. Li, X. F. Yang, M. B. Fan, Y. Q. Wang, and E. Lu, "Vibration response and power flow characteristics of a flexible manipulator with a moving base," Shock and Vibration, vol. 2015, Article ID 589507, 8 pages, 2015.

[10] Z.-C. Qiu, "Adaptive nonlinear vibration control of a Cartesian flexible manipulator driven by a ballscrew mechanism," Mechanical Systems and Signal Processing, vol. 30, pp. 248-266, 2012.

[11] S. S. Ge, T. H. Lee, and J. Q. Gong, "A robust distributed controller of a single-link SCARA/Cartesian smart materials robot," Mechatronics, vol. 9, no. 1, pp. 65-93, 1999.

[12] M. Dadfarnla, N. Jalili, B. Xian, and D. M. Dawson, "A Lyapunov-based piezoelectric controller for flexible cartesian robot manipulators," Journal of Dynamic Systems, Measurement and Control, vol. 126, no. 2, pp. 347-358, 2004.

[13] K. X. Wei, G. Meng, S. Zhou, and J. W. Liu, "Vibration control of variable speed/acceleration rotating beams using smart materials," Journal of Sound and Vibration, vol. 298, no. 4-5, pp. 1150-1158, 2006.

[14] F. W. Liou, A. G. Erdman, and C. S. Lin, "Dynamic analysis of a motor-gear-mechanism system," Mechanism and Machine Theory, vol. 26, no. 3, pp. 239-252, 1991.

[15] U. Andreaus and P. Casini, "Dynamics of friction oscillators excited by a moving base and/or driving force," Journal of Sound and Vibration, vol. 245, no. 4, pp. 685-699, 2001.

[16] H. S. Yan, M. C. Tsai, and M. H. Hsu, "An experimental study of the effects of cam speeds on cam-follower systems," Mechanism and Machine Theory, vol. 31, no. 4, pp. 397-412, 1996.

[17] R.-C. Soong, "A new design method for single DOF mechanical presses with variable speeds and length-adjustable driving links," Mechanism and Machine Theory, vol. 45, no. 3, pp. 496510, 2010.

[18] I. F. Thor and N. Henk, Parametric Resonance in Dynamical Systems, Springer, New York, NY, USA, 2012.

[19] Z. H. Feng and H. Y. Hu, "Principal parametric and three-to-one internal resonances of flexible beams undergoing a large linear motion," Acta Mechanica Sinica, vol. 19, no. 4, pp. 355-364, 2003.

[20] L.-Q. Chen and X.-D. Yang, "Stability in parametric resonance of axially moving viscoelastic beams with time-dependent speed," Journal of Sound and Vibration, vol. 284, no. 3-5, pp. 879-891, 2005. 
[21] X.-D. Yang and L.-Q. Chen, "Stability in parametric resonance of axially accelerating beams constituted by Boltzmann's superposition principle," Journal of Sound and Vibration, vol. 289, no. 1-2, pp. 54-65, 2006.

[22] H. Ding and L. Q. Chen, "Nonlinear dynamics of axially accelerating viscoelastic beams based on differential quadrature," Acta Mechanica Solida Sinica, vol. 22, no. 3, pp. 267-275, 2009.

[23] S.-B. Li and W. Zhang, "Global bifurcations and multi-pulse chaotic dynamics of rectangular thin plate with one-to-one internal resonance," Applied Mathematics and Mechanics, vol. 33, no. 9, pp. 1115-1128, 2012.

[24] M. H. Yao and W. Zhang, "Multi-pulse chaotic motions of high-dimension nonlinear system for a laminated composite piezoelectric rectangular plate," Meccanica, vol. 49, no. 2, pp. 365-392, 2014.

[25] B. Pratiher and S. K. Dwivedy, "Non-linear dynamics of a flexible single link Cartesian manipulator," International Journal of Non-Linear Mechanics, vol. 42, no. 9, pp. 1062-1073, 2007.

[26] D. U. Noiseux, "Measurement of power flow in uniform beams and plates," The Journal of the Acoustical Society of America, vol. 47, no. 1, pp. 238-247, 1970.

[27] J. M. Cuschieri, "Structural power-flow analysis using a mobility approach of an L-shaped plate," Journal of the Acoustical Society of America, vol. 87, no. 3, pp. 1159-1165, 1990.

[28] H. G. D. Goyder and R. G. White, "Vibrational power flow from machines into built-up structures, part I: introduction and approximate analyses of beam and plate-like foundations," Journal of Sound and Vibration, vol. 68, no. 1, pp. 59-75, 1980.

[29] S. J. Walsh and R. G. White, "Measurement of vibrational power transmission in curved beams," Journal of Sound and Vibration, vol. 241, no. 2, pp. 157-183, 2001.

[30] Z. H. Wang, J. T. Xing, and W. G. Price, "An investigation of power flow characteristics of L-shaped plates adopting a substructure approach," Journal of Sound and Vibration, vol. 250, no. 4, pp. 627-648, 2002.

[31] W. J. Choi, Y. P. Xiong, and R. A. Shenoi, "Power flow analysis for a floating sandwich raft isolation system using a higher-order theory," Journal of Sound and Vibration, vol. 319, no. 1-2, pp. 228246, 2009.

[32] X. L. Ma, G. Y. Jin, and Z. G. Liu, "Active structural acoustic control of an elastic cylindrical shell coupled to a two-stage vibration isolation system," International Journal of Mechanical Sciences, vol. 79, pp. 182-194, 2014.

[33] H.-J. Lee and K.-J. Kim, "Multi-dimensional vibration power flow analysis of compressor system mounted in outdoor unit of an air conditioner," Journal of Sound and Vibration, vol. 272, no. 3-5, pp. 607-625, 2004.

[34] P. Coulier, G. Lombaert, and G. Degrande, “The influence of source-receiver interaction on the numerical prediction of railway induced vibrations," Journal of Sound and Vibration, vol. 333, no. 12, pp. 2520-2538, 2014.

[35] M. F. M. Hussein and H. E. M. Hunt, "A power flow method for evaluating vibration from underground railways," Journal of Sound and Vibration, vol. 293, no. 3-5, pp. 667-679, 2006.

[36] S. R. Singiresu, Mechanical Vibration, Pearson Education, 4th edition, 2004.

[37] F. X. Mei, Analytical Mechanics, Beijing Institute of Technology Press, Beijing, China, 2013.

[38] S. O. R. Moheimani and A. J. Fleming, Fundamentals of Piezoelectricity. Piezoelectric Transducers for Vibration Control and Damping, Springer, London, UK, 2006.
[39] D. Gross, W. Hauger, J. Schröder, W. A. Wall, and J. Bonet, Engineering Mechanics 2: Mechanics of Materials, Springer, London, UK, 2011.

[40] X. Q. Wang, W. O. Wong, and L. Cheng, "Modal power flow with application to damage detection," International Journal of Engineering Science, vol. 47, no. 4, pp. 512-523, 2009.

[41] C. C. Liu, F. M. Li, L. Tang, and W. H. Huang, "Vibration control of the finite L-shaped beam structures based on the active and reactive power flow," Science China Physics, Mechanics and Astronomy, vol. 54, no. 2, pp. 310-319, 2011. 

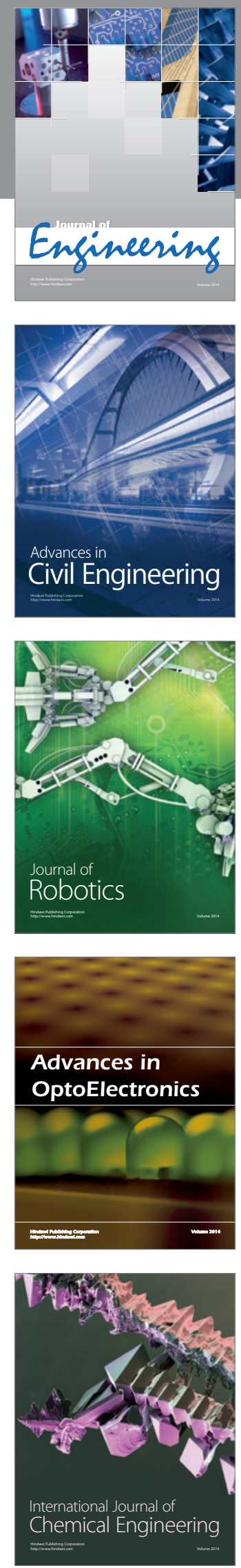

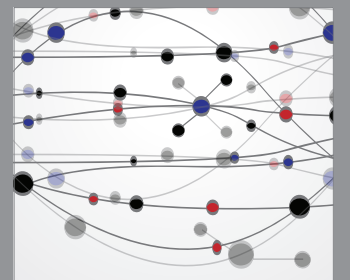

The Scientific World Journal
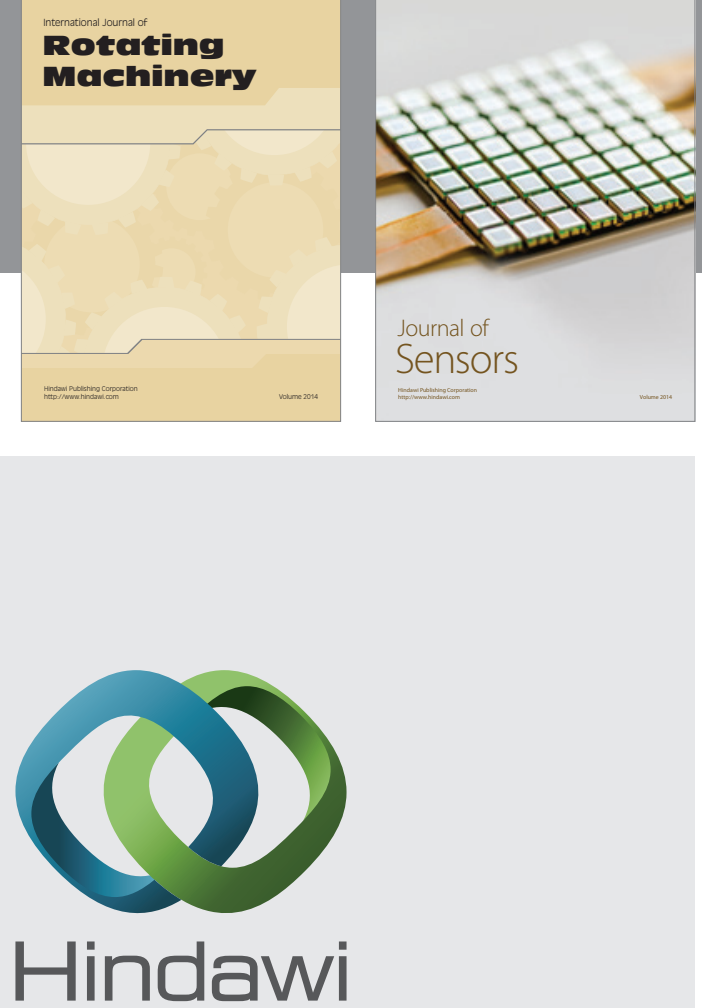

Submit your manuscripts at http://www.hindawi.com
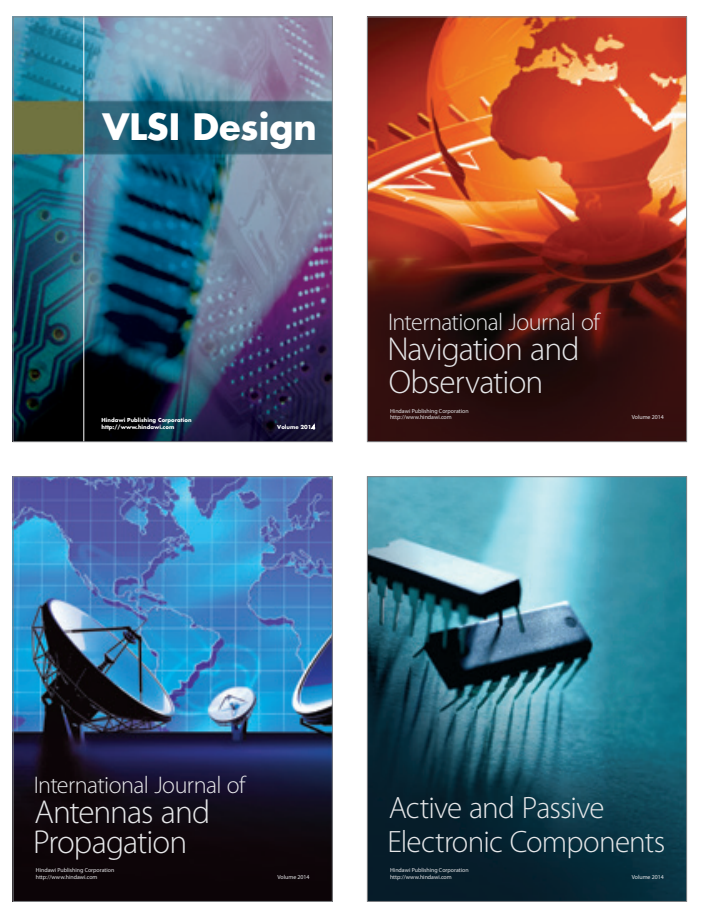
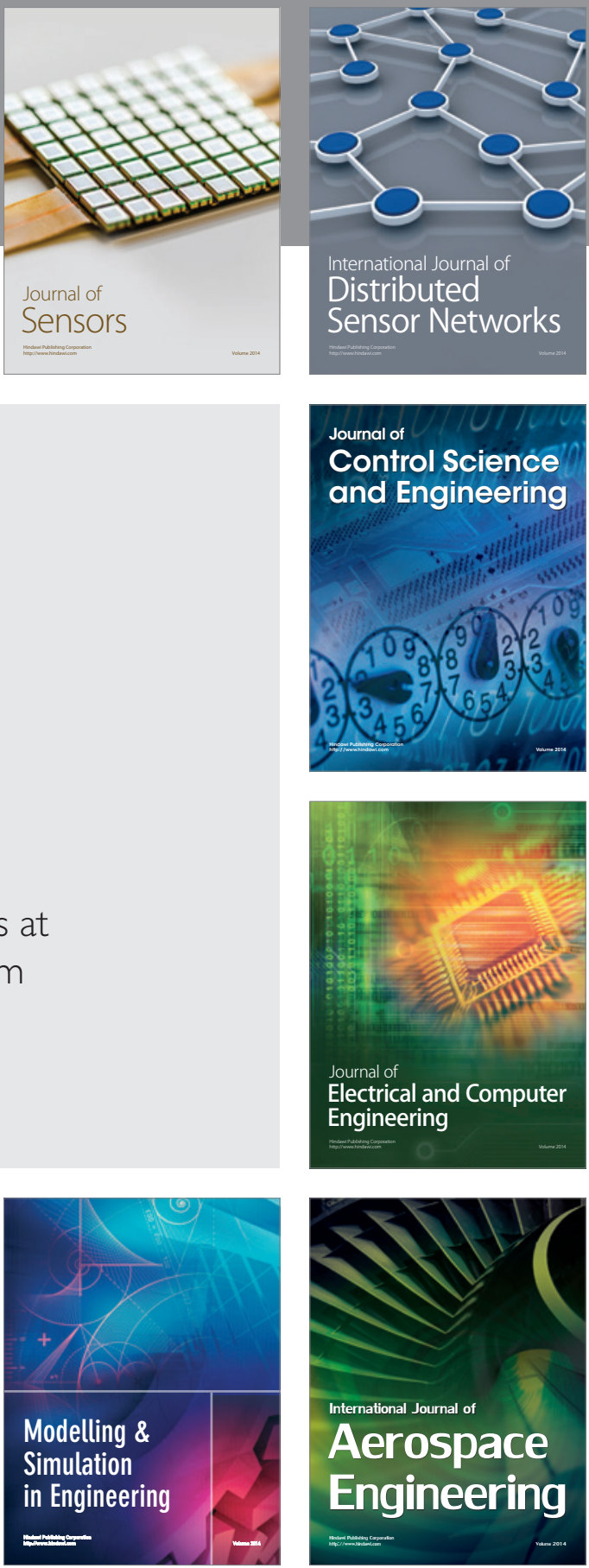

Journal of

Control Science

and Engineering
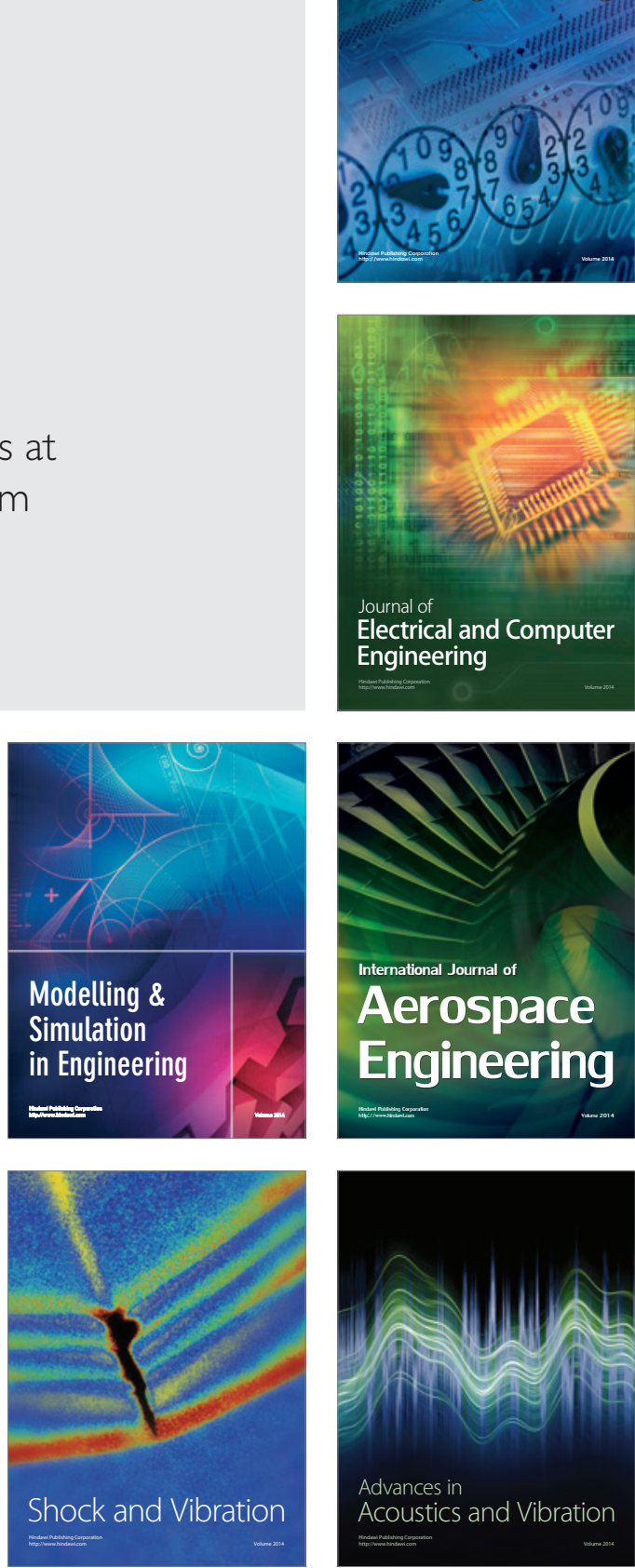